Www.jmscr.igmpublication.org

Impact Factor (SJIF): 6.379

Index Copernicus Value: 79.54

ISSN (e)-2347-176x ISSN (p) 2455-0450

crossrefDOI: https://dx.doi.org/10.18535/jmscr/v6i11.64

Journal Of Medical Science And Clinical Research

\title{
A Study of Oxidative Stress, Antioxidant Status, and Lipid Profile in Type-2 Diabetes Mellitus Patients in Tertiary Care Hospital, Durgapur (West Bengal) India
}

\author{
Authors \\ Syed Khursheed Ejaz ${ }^{1}$, Rajiv Kumar ${ }^{2}$, Chandan Kumar Poddar ${ }^{3}$ \\ ${ }^{1}$ Associate Professor, Department of Biochemistry, Gouri Devi Institute of Medical Sciences and Hospital, \\ Durgapur, West Bengal. \\ ${ }^{2}$ Associate Professor, Department of Pathology, Lord Buddha Koshi Medical College and Hospital, Saharsa, \\ Bihar \\ ${ }^{3}$ Tutor, Department of Microbiology, Lord Buddha Koshi Medical College and Hospital, Saharsa, Bihar \\ Corresponding Author \\ Rajiv Kumar \\ Associate Professor, Dept of Pathology, Lord Buddha Koshi Medical College and Hospital, Saharsa, Bihar
}

\begin{abstract}
Background: Diabetes mellitus is a common endocrine disorder characterized by hyperglycemia, metabolic abnormalities and long term complications afflicting the eyes, kidneys, nerves, and blood vessels.Type-2 Diabetes mellitus (Type-2 DM) is now epidemic in many countries undergoing modernization and industrialization. Diabetes is becoming the third killer of mankind, after cancer and cardiovascular diseases, because of its high prevalence, morbidity and mortality.

Aims and Objectives: The aim of this study was to assess the biochemical parameters in Type-2 Diabetes mellitus patients and normal healthy individuals (Control Group).

Material and Methods

Method: We studied 40 patients with type 2 diabetes and other 40 age-and sex-matched health control. To evaluate the oxidative status we measured the levels of malondialdehyde (MDA) in the serum of all participants. Lipid profile was also estimated through measuring total cholesterol, triglycerides, low-density lipoprotein (LDL), highdensity lipoprotein (HDL) and very low-density lipoprotein (VLDL) levels. Antioxidant levels were also assessed through measuring the serum concentration of uric acid, superoxide dismutase and reduced glutathione (GSH).

Results: The mean values of BSL $(F), B S L(P P), T C, T G$, and LDL were significantly increased $(P<0.001)$ in patients as compared to control. Whereas the mean value of $H D L$ was significantly decreased in patients as compared to control. The Mean value of Malondialdehyde (MDA) was significantly increased $(P<0.001)$ in patients as compared to control The Mean values of SOD, Vitamin $C, E$ were significantly decreased in patients as compared to control.

Conclusion: The findings of this study is in accordance to earlier studies, that there is an increased oxidative stress in diabetics compared to controls; and the oxidative stress further increases as diabetes to cardiovascular diseases. This study emphasizes the importance of assessing the antioxidant status in diabetes in addition to the markers of oxidative stress and lipid profile to formulate the specific therapies for early therapeutic intervention and better treatment of diabetes mellitus. Keywords: Type 2 diabetes mellitus, MDA, Vitamin E, Lipid Profile.

Keywords: Type-2 Diabetes mellitus, hyperglycemia, Insulin resistance, lipid profile, superoxide dismutase, oxidative stress, malondialdehyde, reactive oxygen species.
\end{abstract}




\section{Introduction}

India leads the world with largest number of individuals with diabetes mellitus earning the dubious distinction of being termed the "diabetes capital of the world". It has been estimated that the global burden of type 2 diabetes mellitus (T2DM) will increase to 438 million in 2030. Similarly, for India this increase is estimated to be $58 \%$, from 51 million people in 2010 to 87 million in $2030^{[1,2]}$. Diabetes mellitus is a common endocrine disorder characterized by hyperglycemia, metabolic abnormalities and long term complications afflicting the eyes, kidneys, nerves, and blood vessels. India, a developing country with fast industrialization and a modern lifestyle is facing a grave problem in having the largest number of people with Diabetes which is estimated to reach 80 million by the year $2030 .{ }^{[3,}$

4] Type-2 Diabetes mellitus is the most rapidly growing chronic disease in the world. Diabetes is classified into two types, type-1 DM (Insulin dependent Diabetes mellitus) and type-2 DM (Non insulin dependent Diabetes mellitus). In the early stages, the $\beta$-cells respond to the insulin resistance by secreting increased quantities of insulin and maintain blood sugar at normal levels. But gradually the beta cells begin to fail and the insulin levels decrease, resulting in rising levels of blood sugar. So it is a combination of insulin resistance and beta cell failure that causes Type-2 DM. ${ }^{[5]}$ India is considered the diabetic capital of the world by 2020AD. In India the prevalence is $2-4 \%$ in rural and $4.0-11.6 \%$ in urban areas. Worldwide estimates project that in 2030 the gretest number of individuals with diabetes will be aged 45-64 years. ${ }^{[6]}$ Type-2 DM leads to reduced quality of life and life expectancy, with a greater risk of heary disease, stroke, peipheral neuropathy, renal diseases, cataracts formation, amputation, ketoacidosis. ${ }^{[7]}$ The development of Type-2 DM is caused by a combination of genetic factors related to insulin resistance, impaired insulin secretion and environmental factors such as obesity, lifestyle, mental stress and ageing. ${ }^{[8]}$ Type-2 DM is associated with plasma lipid and lipoprotein abnormalities, including reduced High

Density Lipoprotein(HDL) cholesterol, a predominance of small dense Low Density Lipoprotein (LDL) particles, and elevated Triglycerides (TGS). ${ }^{[9]}$ During the study, serum MDA levels are measured, which is supposed to be an index of lipid peroxidation.

Malondialdehyde is the organic compound with the formula $\mathrm{CH} 2(\mathrm{CHO}) 2$. The structure of this species is more complex than this formula suggests. This reactive species occurs naturally and is a marker for oxidative stress. Malondialdehyde mainly exists in the enol form. ${ }^{[10]}$ Superoxide dismutase (SOD) is dimeric antioxidant enzyme responsible for the quenching of superoxide radicals which are released during the chemical reactions of the various metabolic pathways. Vitamin c (Ascorbic acid) is a six carbon lactone that is synthesized from glucose in the liver of mammalian species but not by humans, human primates and guinea pigs. It acts as chain breaking antioxidant. ${ }^{[11]}$

Vitamin E is a lipid soluble antioxidant present in all cellular membranes protecting against lipid peroxidation. It acts as a chain breaking antioxidant. ${ }^{[12]}$ With this background the present study was examine alterations in biochemical parameters such as blood glucose, lipid profiles, MDA, SOD, vitamin C, vitamin $\mathrm{E}$ in Type -2 diabetic patients in tertiary care Hospital, Durgapur (West Bengal) India.

\section{Materials and Methods}

This study is a cross sectional case-control study, conducted in the Department of Biochemistry in association with Central Pathology Gouri Devi Institute of Medical Sciences and Hospital, Durgapur, West Bengal.

Eighty consented individuals were enrolled in this observational study. They were grouped into two groups, and the study group consisted of 40 patients (22 males and 18 females) with type 2 diabetes mellitus without complications. They were recruited during their routine medical care visits from an outpatient diabetic clinic at Gouri 
Devi Institute of Medical Sciences and Hospital, Durgapur, West Bengal. All patients with diabetes were being treated with stable doses of oral hypoglycaemic agents. The control group consisted of 40 (22 males and 18 females) systemically healthy subjects aged 40-60 years. We the full medical history of all individuals and clinical examinations with laboratory investigations were undertaken to exclude any other systemic and/or local diseases that may affect the parameters examined in this study.

The diagnosis of the patient was done on the basis of the patient's condition, clinical history, personal history, physical examination, laboratory investigations etc. Blood samples were collected twice for fasting \& postprandial BSL.

\section{Inclusion Criteria}

The patients were having Type-2 Diabetes mellitus from January 2017 to January 2018.

\section{Exclusion Criteria}

The patients were having Type-2 DM more than 1 year, and having complications, smokers, and alcoholics are excluded.

\section{Blood Collection}

Informed consent was obtained from all participants. A fasting (12 hours) venous blood sample $(5 \mathrm{ml})$ was drawn from the patients and controls into a sterile disposable syringe which was transferred into centrifuge tubes and was allowed to clot for 30 minutes. The sample was centrifuged at $3000 \mathrm{rpm}$ for 10 minutes and the separated serum was used for the estimation of glucose (GOD-POD method), total cholesterol (CHOD-PAP method), triglycerides (TGL) (GOPPAP method), high density lipoprotein (HDLC) (CHOD-PAP method), low density lipoprotein (LDLC) (Enzymatic Colorimetric Assay), vitamin E (Baker's and Frank's method), Malondialdehyde (MDA) (Thiobarbituric acid) and Ceruloplasmin (copper oxide activity method). Well designed proforma was prepared and patient's history was taken.

\section{Statistical Analysis}

Data were expressed as mean \pm SD. P value $<0.05$ is considered as statistically significant. Statistical analysis was performed using SPSSv10 software package.

\section{Results}

The results of the present study are described in Tables 1-2. The mean age of the control group was $50 \pm 7$ years and for the diabetic group was $53 \pm 4$ years. The fasting blood sugar (FBS) in the diabetic group was $8.24 \pm 0.78 \mathrm{mmol} / \mathrm{l}$ and for the control group was $5.2 \pm 1.2 \mathrm{mmol} / \mathrm{l}$. Based on serum levels of TC and triglycerides, all subjects in the diabetic group were hyperlipidemic (TC > 4.5 and triglyceride $1.7 \mathrm{mmol} / \mathrm{l})$. Subjects in the control group, on the other hand, were normolipidemic $(\mathrm{TC}<4.5$ and triglycerides $<1.7$ $\mathrm{mmol} / \mathrm{l})$. The cholesterol and triglyceride concentrations in serum increased in the diabetic group as compared with the control group ( $\mathrm{p}<$ 0.001). The concentrations of LDL-C and very low-density lipoprotein cholesterol (VLDL-C) were significantly high and the concentrations of HDL-C were significantly low among diabetics (Figures 1). The serum MDA levels, a product of lipid peroxidation, are increased significantly among diabetics (Figure 2). Antioxidant activity was assessed in this study through measuring the concentration of US, SOD and GSH reductase in the serum of diabetic and control groups (Tables 1). The mean values of serum UA of healthy control were $358.36 \pm 38.9$ and $209.9 \pm 28.3$ $\mu \mathrm{mol} / \mathrm{l}$, respectively. In the diabetic group, serum values ranged from 369 to $584 \mu \mathrm{mol} / \mathrm{l}$ with a mean of $461.2 \pm 58.8 \mu \mathrm{mol} / \mathrm{l}$ (Figure 3). Serum GSH activity was significantly low among diabetics; however, Control group (Figures 4 and Table 1). As shown in Table 4, serum TC had significantly strong positive correlation $(r=0.97)$ with serum LDL-C only among diabetics, whereas serum triglycerides had significantly moderate to strong negative correlation with serum HDL-C ( $\mathrm{r}=-$ 0.466) and serum GSH had a positive relation with serum SOD only. 
Table 1: Serum values of lipid profile, MDA, UA, SOD and GSH

\begin{tabular}{|l|c|c|c|c|c|c|c|}
\hline \multirow{2}{*}{ Parameters } & \multicolumn{3}{|c|}{ Diabetics $(\mathrm{n}=40)$} & \multicolumn{3}{c|}{ Control $(\mathrm{n}=40)$} & \multirow{2}{*}{-value } \\
\cline { 2 - 7 } & Mean & Range & SD & Mean & Range & SD & \\
\hline TC $(\mathrm{mmol} / \mathrm{l})$ & 5.14 & $3.62-8.27$ & 1.3 & 3.93 & $2.67-5.8$ & 0.67 & $<0.001$ \\
\hline TG $(\mathrm{mmol} / \mathrm{l})$ & 3.12 & $2.06-4.46$ & 0.67 & 1.67 & $0.54-5.07$ & 1.04 & $<0.001$ \\
\hline HDL-C $(\mathrm{mmol} / \mathrm{l})$ & 0.87 & $0.61-1.7$ & 0.13 & 1.58 & $0.61-2.54$ & 0.52 & $<0.001$ \\
\hline LDL-C $(\mathrm{mmol} / \mathrm{l})$ & 2.74 & $1.16-6.02$ & 1.3 & 1.57 & $0.67-2.58$ & 0.57 & $<0.001$ \\
\hline VLDL-C $(\mathrm{mmol} / \mathrm{l})$ & 1.48 & $0.94-2.05$ & 0.31 & 0.88 & $0.25-1.49$ & 0.38 & $<0.001$ \\
\hline MDA $(\mu \mathrm{mol} / \mathrm{l})$ & 2.39 & $0.85-4.33$ & 0.98 & 1.14 & $0.52-1.86$ & 0.38 & $<0.001$ \\
\hline UA $(\mu \mathrm{mol} / \mathrm{l})$ & 462.16 & $369-588$ & 58.9 & 358.4 & $290.8-428$ & 38.8 & $<0.001$ \\
\hline SOD $(\mathrm{U} / \mathrm{ml})$ & 1.08 & $0.45-1.49$ & 0.54 & 1.48 & $0.78-2.18$ & 0.19 & $<0.001$ \\
\hline GSH $(\mu \mathrm{mol} / \mathrm{l})$ & 2.16 & $0.62-3.48$ & 0.88 & 3.24 & $1.91-4.43$ & 0.8 & $<0.001$ \\
\hline
\end{tabular}

TC, total cholesterol; TG, triglycerides; HDL-C, high-density lipoprotein cholesterol; LDL-C, low-density lipoprotein cholesterol; VLDL-C, very low-density lipoprotein cholesterol; MDA, malondialdehyde; UA, uric acid; SOD, superoxide dismutase; GSH, glutathione.

Table 2: Correlations among serum estimates

\begin{tabular}{|l|c|c|}
\hline Parameter & $\mathrm{R}$ & $\mathrm{p}$-value \\
\hline TC with LDL-C & 0.973 & $<0.001$ \\
\hline TG with HDL-C & -0.467 & $<0.05$ \\
\hline TG with VLDL-C & 1.000 & $<0.001$ \\
\hline HDL-C with VLDL-C & -0.427 & $<0.05$ \\
\hline GSH with SOD & 0.411 & $<0.05$ \\
\hline
\end{tabular}

TC, total cholesterol; TG, triglycerides; HDL-C, high-density lipoprotein cholesterol; LDL-C, low-density lipoprotein cholesterol; VLDL-C, very low-density lipoprotein cholesterol; SOD, superoxide dismutase; GSH, glutathione.

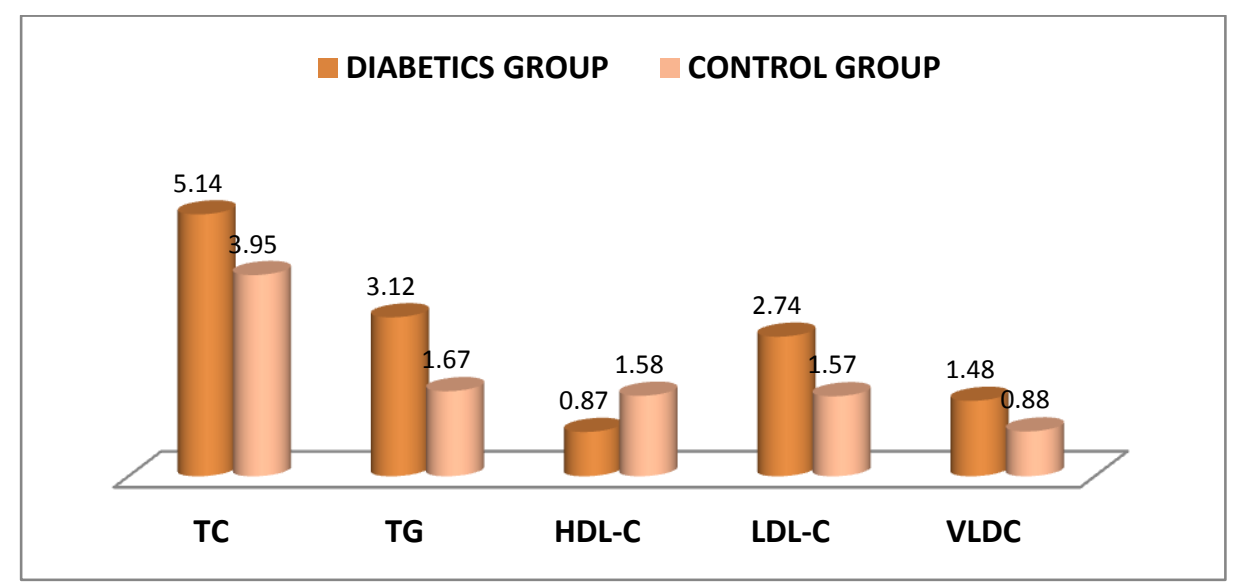

Figure 1: Serum lipid parameters

TC, total cholesterol; TG, triglycerides; HDL-C, high-density lipoprotein cholesterol; LDL-C, low-density lipoprotein cholesterol; VLDL-C, very low-density lipoprotein cholesterol

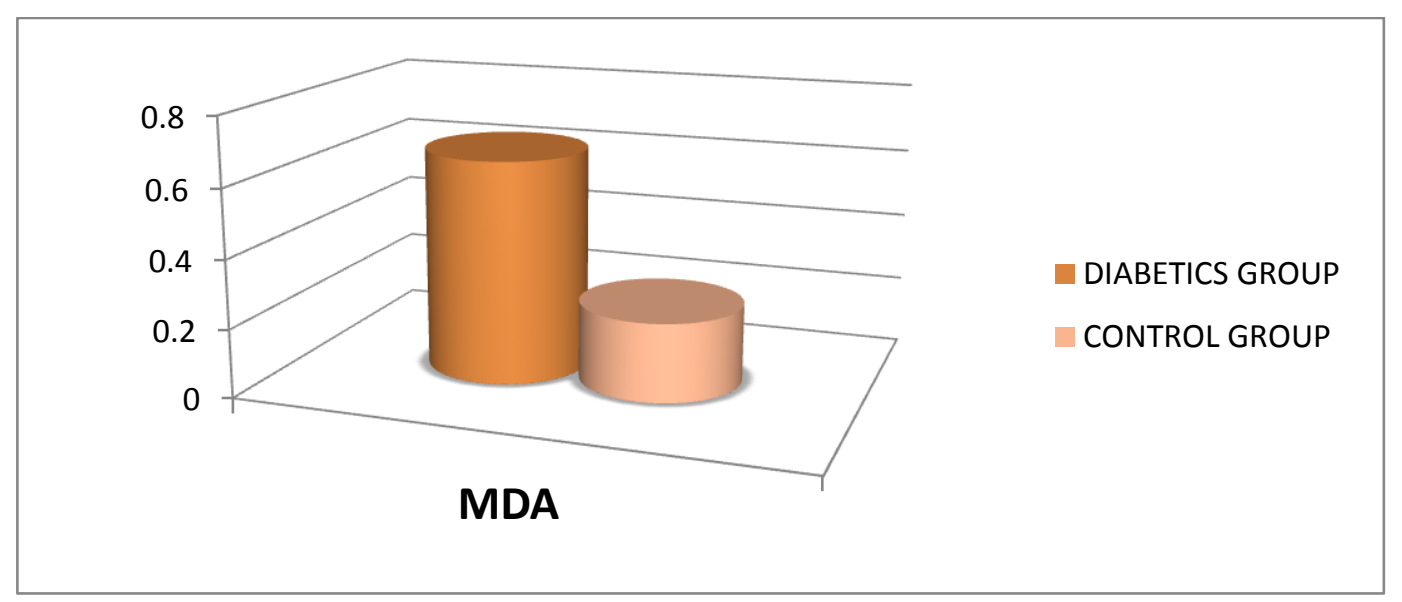

Figure 2: Serum malondialdehyde (MDA) levels 


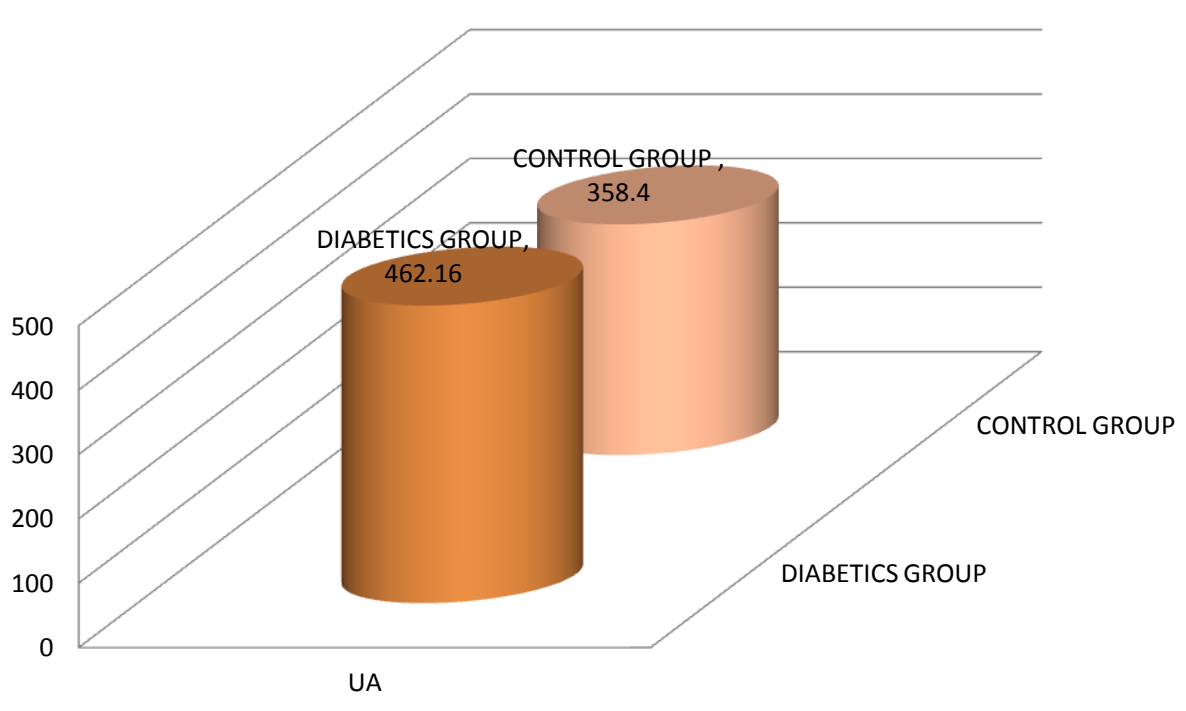

Figure 3. Serum uric acid (UA) levels

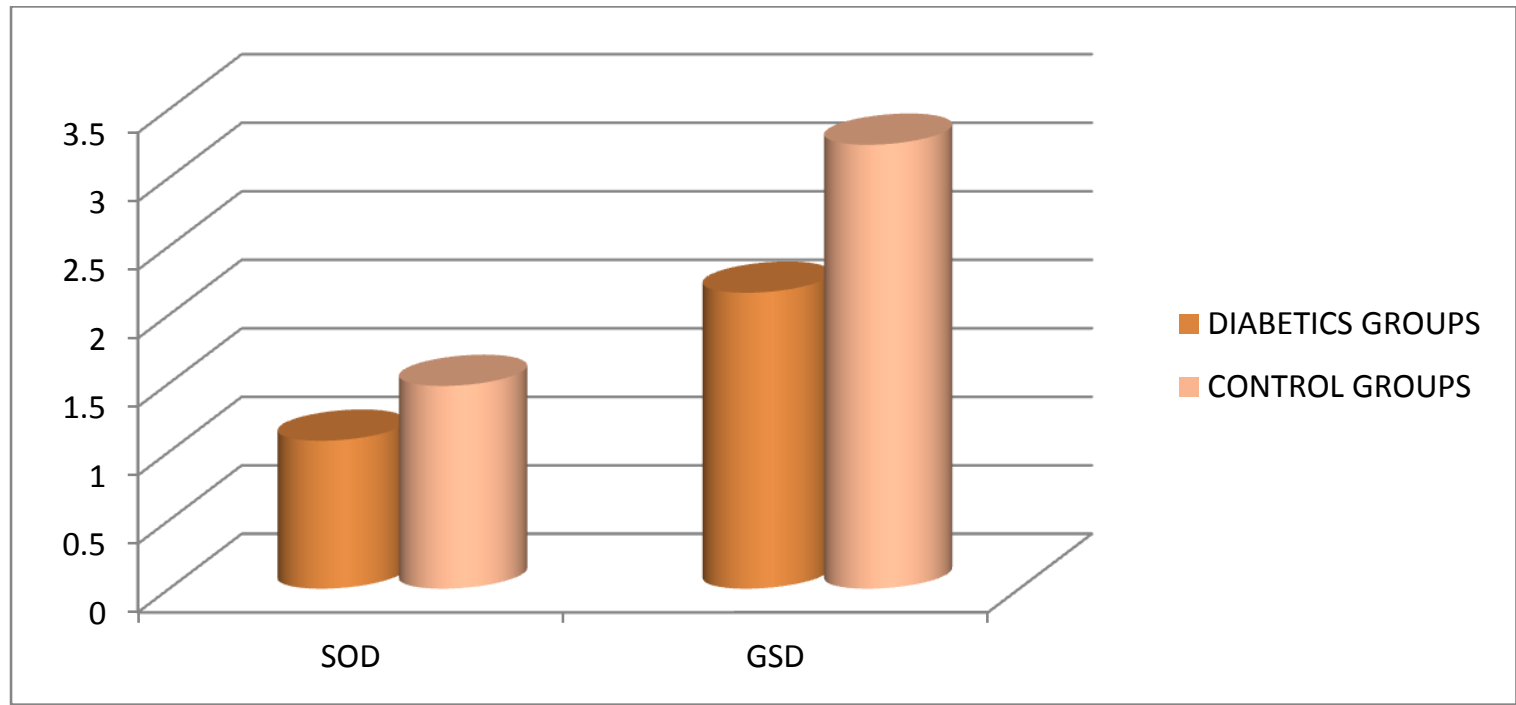

Figure 4: Serum superoxide dismutase (SOD) and glutathione (GSH) levels

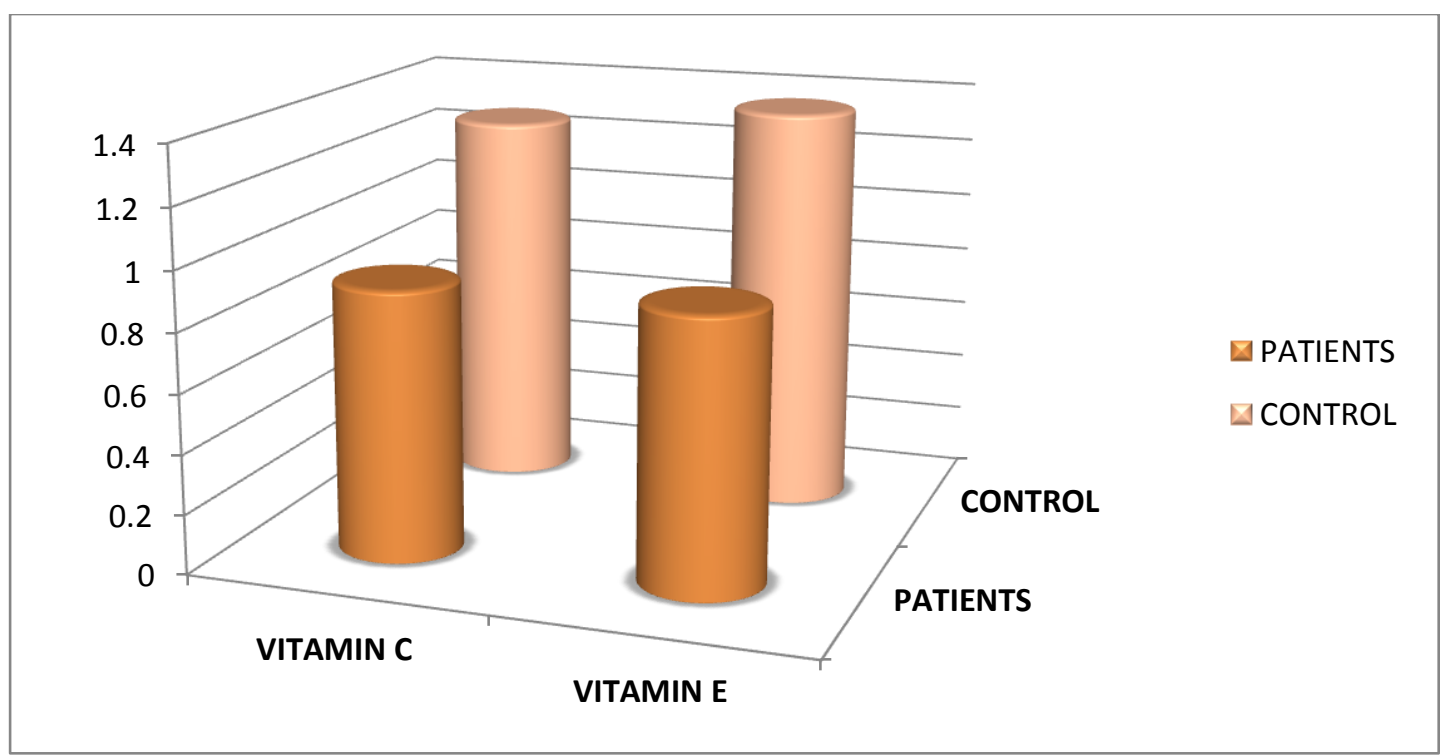

Fig 5: Antioxidants in Patients and Control 


\section{Discussion}

Diabetes mellitus is characterized by chronic hyperglycaemia due to defects in insulin secretion, peripheral insulin action or both which leading to alteration in the fat, proteins and carbohydrate metabolism of the individuals. Several distinct types of diabetes mellitus are caused by complex interactions between genetics and environmental factors. Though great attention was centred on the diabetic complications which had cardiovascular nature, nephropathy, retinopathy and neuropathy, the pulmonary complications of type 2 diabetes mellitus have been poorly characterized. Type 2 Diabetes mellitus is characterised by persistent hyperglycaemia and abnormal metabolisms of carbohydrates, proteins and lipids. ${ }^{[13]}$ Type-2 DM is a heterogenous chronic disorder characterized by hyperglycemia and its complications. Type- 2 DM is the most prevalent variant due to combination of insulin resistance and relative insulin deficiency, due to pancreatic beta cell failure. ${ }^{[14]}$ Impaired insulin secretion is generally progressive, and its progression involves glucose toxicity and lipotoxicity. When untreated, these are known to cause a decrease in pancreatic cell mass. The progression of the impairment of pancreatic cell function greatly affects the long term control of blood glucose. While patients in early stages after disease onset chiefly show an increase in postprandial blood glucose as result of increased insulin resistance and decreased early phase secretion, the progression of the determination of pancreatic cell function subsequently causes permanent elevation of blood glucose. Hyperglycemia leads to disturbances in carbohydrate, lipid and protein metabolism. Every cell in human body with diabetes is exposed to abnormally high glucose levels, but hyperglycemia selectively damage specific cell type, due to the failure in regulation of these cells for glucose uptake. ${ }^{[15]}$ In view of this, we have investigated the biochemical parameters like BSL (F), BSL (PP), lipid profile (TC, TG, HDL, LDL), lipid peroxidation product as MDA, enzymatic antioxidant like SOD, and non enzymatic antioxidants such as vitamin $\mathrm{C}$ and $\mathrm{E}$. We observed significantly increased $(p<0.001) \mathrm{TC}$, TG, LDL levels and decreased HDL levels in patients as compared to control. Our study correlates with previous studies. ${ }^{[16]}$ Insulin resistance may play a vital role in the development of diabetic dyslipidemia, by influencing several factors like genetic, obesity etc. Insulin resistance increases efflux of free fatty acid from adipose tissue and impaired insulin causes decrease uptake of free fatty acid by skeletal muscles, causes fatty acid flux to the liver, kidney etc. In the presence of Insulin resistance, free fatty acid in the form of TG are deposited in muscle, liver, heart, and pancrease. Insulin resistance also increases the hepatic lipase activity, which is responsible for hydrolysis of TG in LDL and HDL particles and leads to smaller and denser LDL particles. ${ }^{[17,18]}$ In the present study MDA level was increased significantly in diabetic patients comparing to healthy subjects. Our results are similar to earlier workers $^{[19]}$ who found increased MDA level in serum of Type -2 DM patients. The rise in the MDA indicated increased oxidative stress, caused by free radical mediated lipid peroxidation in cell membrane. Therefore MDA is a good indicator for oxidative stress. We observed relatively decreased activity of SOD enzyme in patients compared to control. We evaluate antioxidant status by measuring the activity of the antioxidant enzyme like SOD in patients with Type-2 DM and in a healthy control group. A result of our study indicates the action of antioxidant enzyme SOD in diabetic patients which is a sign of oxidative stress. The mean erythrocyte SOD activity in Type-2 DM patients was significantly low as compared to normal control. Similar findings of decreased activity of SOD have been reported by other authors ${ }^{[20,19]}$. hey suggest that there is an imbalance between plasma oxidant and antioxidant system in patients with Type-2 DM. The autooxidation of glucose results in the formation of hydrogen peroxide ( $\mathrm{H} 2 \mathrm{O} 2)$ which decrease the activity of SOD. The activity of SOD decrease in erythrocytes of diabetic subjects duet 
to glycation of SOD enzyme. In the present study decreased activity of SOD in the erythrocytes appears due to the change of protein synthesizing machinery in the erythrocyte. Diminished activity of SOD results into increased oxidative stress. In our study the lower levels of vitamin $\mathrm{C}$ and $\mathrm{E}$ are seen in type-2 DM patients. Our results are similar to other researchers ${ }^{[21]}$. Vitamin $\mathrm{C}$ and $\mathrm{E}$ are diet derived and detoxify free radicals by chain breaking reaction. They also interact in recycling processes to generate reduced forms of the vitamins which may destroy free radicals.

The diagnostic difficulties encountered were parallel to those experienced by different authors working on similar projects- Some studies suggest that tuberculosis can even cause diabetes in those not previously known to be diabetic. Many studies have used oral glucose tolerance testing to show that patients with tuberculosis have higher rates of glucose intolerance than community controls. ${ }^{[22,}$ 22,24] Salmonella was first recognised in patients with underlying condition such as diabetes mellitus. Salmonella has been rarely cited as significant higher rates of glucose intolerance in this population. ${ }^{[25,26]}$ A large number of studies report an increased risk for Type 2 diabetes (T2D) in patients with chronic $\mathrm{HCV}$ infection. ${ }^{[27,28]}$

\section{Conclusion}

In our study there is a disturbed lipid profile in Type-2 DM patients. Serum total cholesterol, Triglycerides, LDL, level found increased while HDL level decreased. Hence, high levels of TC, TG, LDL and low levels of HDL may be due to insulin resistance and other influencing factors like obesity, increase calorie intake, genetic, sedentary lifestyle, lack of muscular activity, in type-2 DM patients. In our study increased MDA levels indicate the increased oxidative stress. Enzymatic antioxidant like SOD activity is significantly decreased in our study. Non enzymatic antioxifdant vitamin $\mathrm{C}$ and $\mathrm{E}$ levels are also decreased. From this we can conclude that decreased erythrocyte SOD activity, decreased vitamin $\mathrm{C}$ and $\mathrm{E}$ levels indicate the reduced antioxidant status in Type-2 DM patients. Hence dietary supplementation of vitamin $\mathrm{C}$ and $\mathrm{E}$ may be helpful to improve antioxidant status of the body.

\section{References}

1. V. Mohan, S. Sandeep, R. Deepa, B. Shah \& C. Varghese, Epidemiology of type 2 diabetes: Indian scenario. Diabetes Indian Journal of Medical Research. 2007; 125: 217-230.

2. Sunitha S, Gandham Rajeev, Wilma Delphine Silvia CR and Soniya Rao, Evaluation of Significance of Liver Enzymes as Screening Tests for the Early Detection of Clinically Asymptomatic Non Alcoholic Fatty Liver Disease in Type 2 Diabetes Mellitus Patients. International Journal of Biomedical and Advance Research 2015; 6(12): 860-863.

3. Bjork S, Kapur A, King H, Nair J, Ramchandran A. Global policy :Aspects of diabetes in India. Health policy 2003; 66:6172.

4. Rao MB, Prasek M, Metelko Z. Organisation of diabetes health care in Indian rural areas. Diabetes Croatica2002; 31(3):161-171.

5. Kumawat M, Pahwa MB, Gahlaut VS, Singh N. Status of Antioxidant Enzymes and Lipid Peroxidation in Type 2 Diabetes Mellitus with Micro Vascular Complications. The open endo J2009; 3:12-5.

6. Songa RM, Siddharth K, Sudhakar K. Lipid Profile in Type-2 Diabetes Mellitus with obesity. Bulletin of Pharmaceutical \& Medical Sciences.2013; 1;2.

7. Masur K, Thevenod F, Zanker KS (eds). Pathophysiology of Diabetes Mellitus Type 2: Roles of Obesity, Insulin Resistance and $\beta$ cell Dysfunction. Diabetes and cancer. Epidemological Evidence and Molecular Links. Front Diabetes.Basel, Karger20008; 19:1-18. 
8. Genomics, type 2 diabetes, and obesity. [N Engl J Med. 2010]

9. American Diabetes Association: Management of dyslipidemia in adults with diabetes (Position Statement). Diabetes Care 26 (Suppl. 1):S83-S86, 2003.

10. V. Nair, C. L. O'Neil, P. G. Wang (2008) Malondialdehyde. Ncyclopedia of Reagents for Organic Synthesis, John Wiley and Sons New York,

11. Pujari KN, Jadkar SP, et al. Variations in Vitamin $C$ levels in Leukemias. Biomedical Research2011; 23(2): 307-311.

12. Pujari KN, Jadkar SP, Zende PD, Kulkarni A, Tuljapurkar VB. Quantitative Variation of Vitamin $E$ levels in Leukemias. IJBPS2012; 2(3):47-52.

13. Ejaz SK, Hasan AR, Poddar CK, et al. A comparative study on pulmonary function test in type II diabetics and non-diabetics in a tertiary care hospital in Koshi region (Northern Bihar), India. J. Evid. Based Med. Healthc. 2018; 5(30), 2199-2203. DOI: $10.18410 /$ jebmh/2018/455.

14. Habib P, Sarah S. Peroxidase Activity and Other Biochemical Parameters in Female with Type 2Diabetes Miletus with and without Coronary Arteriosclerosis. International Jornal of Applied Sciences and Technology2014;4(3)

15. Ronald M. Krauss, MD, Lipids and Lipoproteins in Patients With Type 2 Diabetes, Diabetes Care June 2004;27: 6 14961504

16. Maharjan BR, Jha JC, Adhikari D, Vishwanath P, Baxi J, Alurkar VM, Singh PP. A study of oxidative stress, antioxidant status and lipid profile in diabetic patient in the western region of Nepal. Kathmandu University Medical Journal2008; 6(1):16-22.

17. Venkatesh R, Kalaivani K. Lipid Profile Changes in Type-2 Diabetes mellitus. International Journal of Pharmaceutical
Research \& development.2013;5(07):3539.

18. Rajprabha, Hamid A, Meena RK, Syedyawer H, Study of Antioxidant enzyme-Superoxide Dismutase Activity and Lipid Profile in Diabetes Mellitus patients. IJHBR2014; 2:22-9.

19. Suryawanshi NP, Bhutey AK, Nagdeote AA, Jadhav, Manoorkar GS. Study of Lipid Peroxide and Lipid Profile in Diabetes Mellitus.Ind Journal of Clinical Biochemistry2006; 21(1):126-30. 6.

20. Kumawat M, Pahwa MB, Gahlaut VS, Singh N. Status of Antioxidant Enzymes and Lipid Peroxidation in Type 2 Diabetes Mellitus with Micro Vascular Complications. The open endo J2009; 3:12-5

21. Martini (2004) Fundamentals of Anatomy and Physiology. 6th edn Benjamin Cummings, San Francisco.

22. Abbras CK. Fc receptor-mediated phagocytosis: abnormalities associated with diabetesmellitus. Clin Immunol Immunopathol 1991;58:1-17. [PubMed: 1983966]

23. Ahmad I, Mishra A, Poddar CK, et al. Comparison of Ziehl-Neelsen stains with fine needle aspiration technique in diagnosis of tuberculous lymphadenitis in tertiary care hospital, South Bihar (India). J Evolution Med Dent Sci 2017;6(63):4598-4602.

24. Singh MN, Lal A, Poddar CK, et al. Efficacy of fineneedle aspiration technique, Ziehl-Neelsen stains and culture (BACTEC) in diagnosis of tuberculous lymphadenitis in tertiary care hospital, Gaya, India. J Evid Based Med Healthc 2017;4(70):4164-4169.

25. Jacobs JL, Gold JW, Murray HW, et al. Salmonella infections in patients with the acquired immunodeficiency syndrome. Ann Intern Med 1985;102(2):186-188.

26. Ejaz SK, Poddar CK, Singh MN, et al. Antimicrobial susceptibility of salmonella 
enterica serovars in a tertiary care hospital in Koshi region (Northern Bihar), India. J Evolution Med Dent Sci 2017;6(22):18081811.

27. White DL, Ratziu V, El-Serag HB. Hepatitis $\mathrm{C}$ infection and risk of diabetes: a systematic review and meta-analysis. $\mathrm{J}$ Hepatol (2008) 49(5):831-44. doi:10.1016/j.jhep.2008.08.006.

28. Singh MN, Lal A, Poddar CK, et al. Comparative evaluation of ELISA and rapid screening techniques for the diagnosis of $\mathrm{HCV}$ in haemodialysis patients in tertiary care hospital, South Bihar, India. J Evolution Med Dent Sci 2017; 6(93):6683-6687. 